\title{
SPONTANEOUS INTRAMURAL SMALL BOWEL HEMATOMA INDUCED BY ANTICOAGULANT THERAPY: REVIEW AND CASE REPORT
}

Mauricio P. Sorbello, Edivaldo M. Utiyama, José G. Parreira, Dario Birolini, Samir Rasslan

\section{INTRODUCTION}

Abdominal pain accounts for 5-10\% of all cases assessed in hospital emergency services. Of these, only 20 to $25 \%$ will require admittance to a hospital or surgery. In approximately $40 \%$, of these cases, the diagnosis is uncertain. ${ }^{1}$

Of particular note among the causes of abdominal pain that necessitate surgery are appendicitis and intestinal obstruction. The most frequent causes of obstruction are adhesions $(60 \%)$, hernias (15\%), neoplasia $(6 \%)$ and rare causes $(6 \%){ }^{2}$ In many cases, surgery comes to represent both a diagnostic and therapeutic measure. In some cases, however, we may be confronted by an obstructive condition in which more conservative measures are recommended.

The aim of this article is to highlight the rare occurrence of acute abdominal obstruction due to spontaneous intramural small bowel hematoma resulting from the use of oral anticoagulants by reporting on a case attended at the General Surgery Service of the Clinical Surgery Division III of the Hospital das Clínicas - University of São Paulo Medical School (HC - FMUSP), as well as presenting a systematic review of the literature from the last 25 years.

\section{CASE DESCRIPTION}

The case concerns a 59 year old male patient, a trader from Sao Paulo, with a 28-year history of dyslipidemia, arterial hypertension, congestive heart failure, and acute myocardial infarction who was using captopril $75 \mathrm{mg}$ /day, carvedilol $50 \mathrm{mg} /$ day, digoxin $0.25 \mathrm{mg} /$ day, spirolactone $25 \mathrm{mg} /$ day, simvastatin $40 \mathrm{mg} /$ day, acetylsalicylic acid $200 \mathrm{mg} /$ day and amiodarone $200 \mathrm{mg} /$ day. As a sufferer of chronic atrial fibrillation, the patient used an implantable

Hospital das Clínicas da Faculdade de Medicina da Universidade de São Paulo

Email: mausorbello@hotmail.com cardioverter-defibrillator and consequently required oral anticoagulant therapy with sodium warfarin (Marevan ${ }^{\circledR}$ $5 \mathrm{mg} /$ day), initiated one month prior to presentation.

When admitted to the emergency service on June $5^{\text {th }}$, 2006, the patient was complaining of a diffuse abdominal pain that had begun two days earlier. It was more pronounced on the left side and was progressively worsening; only slight relief from the symptoms was obtained with the use of pain relief drugs. The patient referred to an absence of flatulence and feces during the previous 24 hours, associated with nausea without vomiting. Laboratory examinations confirmed the presence of hematuria.

Physical examination showed the patient to be dehydrated and hemodynamically stable. Examination of the abdomen indicated distension and pain upon palpitation, mainly on the left side, without signs of peritoneal irritation. Rectal examination showed no signs of bleeding. The laboratory findings were the following: hemoglobin $(\mathrm{Hb})$ $13 \mathrm{mg} / \mathrm{dL}$, hematocrit $38.5 \%$, leukocytes $12.100 / \mathrm{mm}^{3}$, no left deviation, activated partial thromboplastin time (APTT) 134 seconds, with relation (R) at 5; and prothrombin time (PT) over 100 seconds - international normalized ratio (INR) greater than 12. Amylase, lipase, arterial gasometry, renal function and electrolytes were normal. Radiographs of the abdomen in the orthostatic, supine and prone positions suggested intestinal obstruction without pneumoperitoneum (Fig.1).

As the diagnostic hypothesis was small bowel hematoma, clinical treatment consisted of gastric decompression, volemic restitution and correction of the coagulopathy with fresh frozen plasma and parenteral vitamin K. On June $6^{\text {th }}$, 2006, the patient was submitted to helical multislice computerized tomography (CT) of the abdomen, which showed segmental thickening of the left side of the jejunum wall, measuring up to $1.2 \mathrm{~cm}$ in thickness and resulting in obstruction of the intestinal passage, together with densification of the adjacent fatty planes, good contrasting of the mesenterial vascular branches and a moderate quantity of liquid in the cavity (Figs. 2 and 3). 
On the same day, the patient presented an episode of melena and extensive painful ecchymosis on the upper limbs.

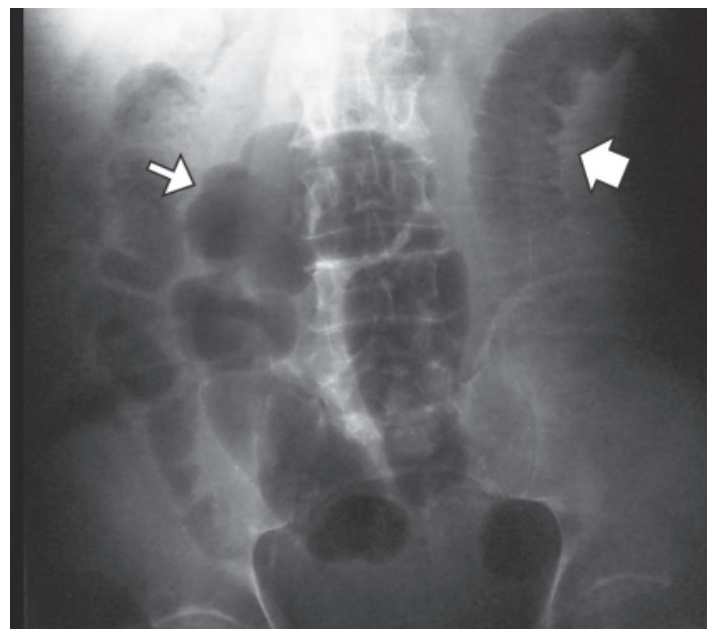

Figure 1 - Radiograph of the abdomen in the supine position, showing the distension of the jejunum flaps (narrow arrow) and "pile of the coins" sign (wide arrow) - both signs of intestinal obstruction.

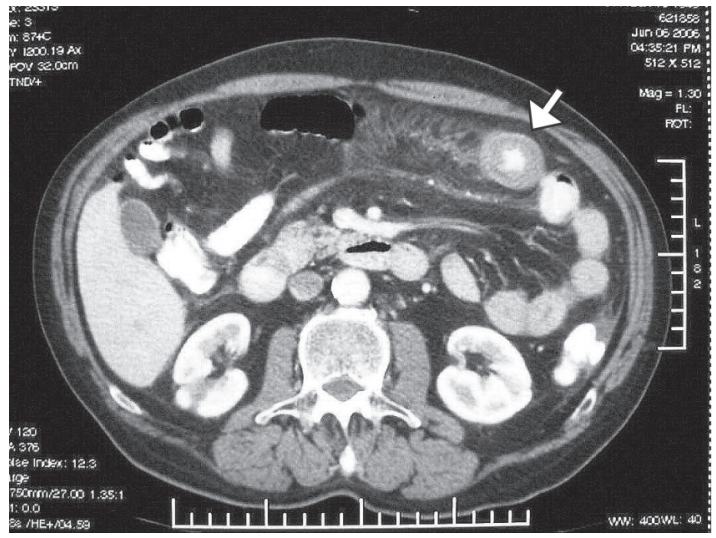

Figure 2 - Contrast CT with transverse slice showing circumferential thickening of the jejunum, known as the "pseudo-kidney" sign (arrow).

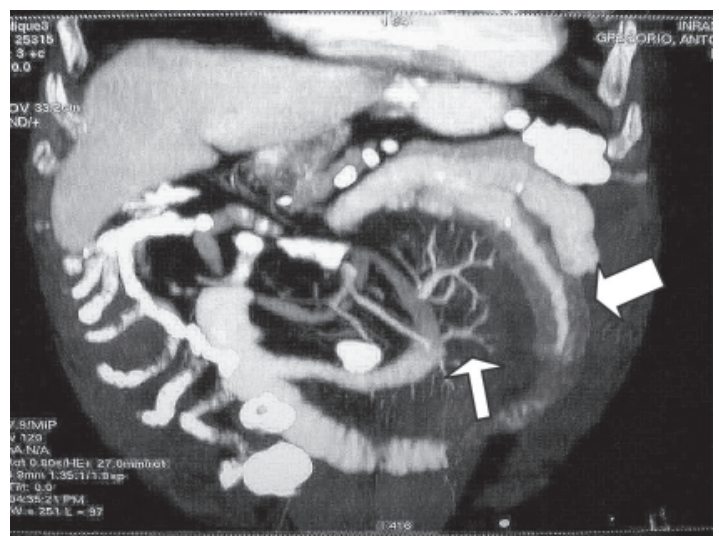

Figure 3 - Contrast CT in a coronal slice, showing thickening of the jejunum wall, "coiled spring" sign (wide arrow) and contrasted mesenteric vascular branches (narrow arrow).
Following initiation of the therapy, the patient showed signs of improvement. On the fourth day, he was given food orally. On June $23^{\text {rd }}, 2006,2.5 \mathrm{mg}$ /day sodium warfarin treatment was reinitiated on alternate days, with adjustment of the dose during hospitalization, in combination with sodium dalteparin (5.000UI daily). On June $26^{\text {th }}, 2006$ the patient was discharged from the hospital.

\section{LITERATURE REVIEW}

The MEDLINE, COCHRANE, LILACS and SciELO databases were used in order to review the literature from the previous 25 years.

The inclusion criteria were:

1) Single or multiple spontaneous small bowel hematoma, exclusive or associated with other areas of bleeding;

2) Patients using vitamin $K$ antagonist oral anticoagulants;

3) Presumptive diagnosis by clinical laboratorial findings, with or without documentation for image examination, in those cases that received clinical treatment;

4) Macroscopical diagnosis through inventory of the cavity or anatomical-pathological documentation in those submitted to surgical treatment.

Cases were excluded where there was a history of trauma, the use of other forms of anticoagulation (even when used in combination with oral anticoagulation), and where hematoma was present in a condition in which the small bowel was not involved. The evaluated variables were: age and sex, clinical manifestations and length of history, hemoglobin levels and prothrombin time at admission, motive and duration of oral anticoagulation therapy, occurrence of digestive hemorrhage, diagnostic strategy, presence of small bowel hematoma, the need for surgical intervention, associated abdominal findings, and time necessary to resolve the condition.

\section{CRITICAL ANALYSIS}

A total of 28 articles were selected, covering the period from 1991 to 2006. Of these, four were excluded for not having been found full text or abstract in the researched databases. An additional three consisted only of abstracts, which was sufficient cause for their use as case reports, but not in the analysis of variables ${ }^{3,4,5}$, this encompassed a total of 57 published cases.

The incidence was higher in males (60\%). The average age, described in 40 cases, was 57.6 years $(32-78$ years). Upon admission to the emergency service, the most frequent clinical condition, also described in 40 cases, was abdominal pain, which was present in $97.5 \%$ of the cases, 
with nausea associated with half of these cases, and vomiting in an additional 40\%; these latter two items are related to high obstructions involving the duodenum and proximal jejunum. Abdominal pain was the only symptom in $20 \%$ of cases, and irradiation in the lumbar region was mentioned in one case, mimicking renal colic. ${ }^{6}$ The obstructive condition, characterized by abdominal distension, halted evacuation and elimination of flatulence in $45 \%$ of cases, all involving either jejunum or ileum and one including the ileocecal valve. ${ }^{7}$ Some cases were related to extraintestinal manifestations, among them: epistaxis, hematuria, ${ }^{8}$ ecchymosis in the upper limbs and face, ${ }^{9}$ conjunctival hemorrhage ${ }^{10}$ and mild acute pancreatitis ${ }^{11}$ (Table 1).

Table 1 - Signs and symptoms present upon admission in 40 patients diagnosed with spontaneous small bowel hematoma due to the use of oral anticoagulation.

\begin{tabular}{lcc}
\hline Signs and symptoms & N* & $\%$ \\
\hline Abdominal pain & 39 & 97.5 \\
Nausea & 20 & 50 \\
Intestinal obstruction & 18 & 45 \\
Vomiting & 16 & 40 \\
Fever & 2 & 5 \\
Anorexia & 2 & 5 \\
Epistaxis & 2 & 5 \\
Hematuria & 2 & 5 \\
Ecchymosis1 & 2.5 & \\
Conjunctival hemorrhage1 & 2.5 & \\
\hline
\end{tabular}

Source: 21 selected articles and case reports were reviewed

The incidence of digestive hemorrhage was $42.4 \%$, which was exteriorized in the form of melena in $50 \%$ of cases, hematemesis associated with melena in $21.4 \%$, only hematemesis in $21.4 \%$, and hematochezia in $7.1 \%$ of these cases. The average time from the appearance of symptoms until medical attendance was 2.5 days ( 3 hours-5 days). The reason for the use of oral anticoagulant was described in 43 cases. Atrial fibrillation was responsible in $23.3 \%$ of cases, followed by prosthetic heart valve $(20.9 \%)$ and deep vein thrombosis (13.9\%), as shown in Table 2. The duration of anticoagulant therapy was evaluated in 30 cases, and the average was 25.4 months (18 days - 84 months). There is one case described as "longer than 3 months" and another "dose increased ten days ago". ${ }^{10}$ In laboratory tests, the average $\mathrm{Hb}$ was 11.5 (5.8-14.3). In one case, it was described as "normal", ${ }^{12}$ and in another, the number of erythrocytes was within the reference value. ${ }^{8}$

In order to present the results more clearly, we separated them into two periods, pre- and post-1991.

Until 1991, the results for PT were described in percentage terms. The observed average was $16.8 \%$ (5-80\%). It was
Table 2 - Reason for oral anticoagulation

\begin{tabular}{lcc}
\hline & $\mathrm{N}$ & $\%$ \\
\hline Atrial fibrillation & 10 & 23.3 \\
Prosthetic heart valve & 9 & 20.9 \\
Deep vein thrombosis (DVT) & 6 & 13.9 \\
Chronic arterial obstruction (CAO) & 4 & 9.3 \\
Pulmonary thromboembolism (PTE) & 4 & 9.3 \\
Cerebral vascular event & 2 & 4.7 \\
Correction of aortic aneurism & 2 & 4.7 \\
Ischemic coronary disease (ICD) & 2 & 4.7 \\
ICD and CAO & 1 & 2.3 \\
ICD and cardiac arrhythmia & 1 & 2.3 \\
DVT and PTE & 1 & 2.3 \\
ICD and arthritis & 1 & 2.3 \\
\hline TOTAL & 43 & 100 \\
\hline
\end{tabular}

Source: 21 selected articles and case reports were reviewed

described as "lower than $15 \% "$ "(8) in two cases and "lower than 5\%" in an additional three cases ${ }^{(13)}$. After this period, the values are expressed using the INR. The average obtained was 7.5 (1.1 - 18.4). In two cases, it was "undetectable", 14,15 one result was "greater than 12", and another two cases were reported PT as 50.2 and 49.7 seconds. ${ }^{10,16}$

With regard to diagnostic strategies, there are no reports of the use of CT or ultrasound (US) until 1986. Hence, we will present the results of these first five years of research, followed by the last 20 years, when CT and US were available. In the first period, diagnoses were based on clinical and laboratory findings in $33 \%$ of cases. Simple radiography was performed in $6.7 \%$ of cases, and this was complemented with contrast radiography of the intestinal transit in $20 \%$ of cases. Intraoperative diagnosis occurred in $40 \%$ of cases, and laparoscopy was used in one case. ${ }^{8}$

Starting in 1987, there was a reduction in the number of surgically based diagnoses; such operations were performed without previous exams in only three $(9.7 \%)$ out of 31 cases. In fact, it is at this time when CT was adopted as the primary method. Of these three surgeries, sectioning of the intestinal flap was performed in two because of the presence of ischemia and the non-viability of the segment, probably a result of the compressive effect of the hematoma, which impaired local blood irrigation.

The use of US as the only complementary exam occurred in $6.45 \%$ of cases, and CT in $54.8 \%$ of cases. CT alone or in conjunction with other methods, such as US and endoscopy, was responsible for $92 \%$ of the diagnoses (Table 3).

Surgery was indicated in distinct situations related to two distinct two periods. From 1981 to 1986, it was used in $40 \%$ of cases, always as a diagnostic method. In the second period (1987-2006), it was performed in $28 \%$ of the cases; as a therapeutic measure in $67 \%$ of these cases. 
Table 3 - Diagnostic strategies after 1986

\begin{tabular}{lcc}
\hline Complementary diagnostic method & $\mathrm{N}$ & $\%$ \\
\hline Computerized tomography & 17 & 54.8 \\
Computerized tomography + US & 8 & 25.8 \\
Intraoperatoive & 3 & 9.7 \\
Ultrasound & 2 & 6.5 \\
Computerized tomography + GE & 1 & 3.2 \\
\hline TOTAL & 31 & 100
\end{tabular}

US=ultrasound; GE=gastrointestinal endoscopy

Source: 21 selected articles and case reports were reviewed

Regarding the location of the hematoma in the intestinal wall, the jejunum was the most affected (71.6\%), followed by the duodenum (29.8\%) and the ileum (15.8\%).

The most frequently reported intra-operational findings while inventorying the cavity were: hemoperitoneum $(10.3 \%)$, necrosis of the intestinal flap (10.3\%), hematoma of mesenterium (10.3\%), hemoretroperitoneum (5.1\%) and cecal hematoma $(2.6 \%)$.

The resolution time of the condition as shown by intestinal transit and the acceptance of oral alimentation, described in 12 cases, was 10.1 days (4-16 days). There is only one report of a late complication, which was described as stenosis and diagnosed after three weeks through barium x-ray. ${ }^{8}$

\section{DISCUSSION}

The first report of intramural intestinal hematoma was given by McLouchlan ${ }^{16}$ in 1838, following an autopsy on a man whose cause of death was recorded as "dehydration and bowel obstruction," attributed to a "false aneurysm." The first radiological description occurred more than one hundred years later, when Liverud reported a case involving the jejunum. ${ }^{17}$

This condition rarely occurs spontaneously and is more frequently associated with abdominal trauma. Among nontraumatic causes, the use of oral anticoagulants is the main etiological factor. ${ }^{18}$ The only reference to the estimated incidence of spontaneous small bowel hematoma related to anticoagulation is that it affects 1:2500 anticoagulation patients/year in research carried out in Switzerland. ${ }^{19}$ In this study, both the use of an oral anticoagulant and heparin are named as causes. With regard to the exclusive use of heparin as a cause of the condition, its incidence is even lower and has been the motive for few reports in the literature. ${ }^{19-21}$

Despite its rarity, the number of reported cases has increased, both because of the availability of current imagebased diagnostic methods as well as the growing number of patients on anticoagulation therapy. Other known causes are hemophilia, Von Willebrand disease, idiopathic thrombocytopenic purpura, lymphoproliferative diseases, ${ }^{22}$ collagenosis, vasculopathies and peptic ulcer. Pancreatic diseases, such as pancreatitus, can be either a cause ${ }^{23}$ or a consequence. ${ }^{16}$

The small bowel is affected in up to $85 \%$ of the occurrences of spontaneous intramural gastro-intestinal tract hematoma caused by the use of oral anticoagulation, with the jejunum being the most affected region, in contrast with the post-traumatic findings, which affect the duodenum more.$^{18}$

We also found reports of spontaneous hematoma in the mesenterium, retroperitoneum and abdominal wall. ${ }^{24}$ More severe cases may take the form of acute abdomen with necrosis of the flap, requiring emergency surgery. ${ }^{7}$ In the Brazilian literature, we found three cases ${ }^{9,16,25}$ described in the last 44 years; one occurred in the jejunum (and was diagnosed following necropsy in a patient with a previous history of ischemic stroke), and two occurred in the duodenum. In one of these, obstruction of the Vater's papilla resulting from the hematoma led to a mild acute pancreatic complication. ${ }^{16}$

Hemorrhaging originates in the submucosa, and, although it has not been proven by other studies, it is believed that the progression of the symptoms is due to intramural osmotic gradient and the presence of the hematoma, leading to an expansion of the intestinal wall. ${ }^{26}$

As shown in our results, clinical suspicion is fundamental in making the diagnosis. The clinical condition may vary according to the location of the hematoma, and symptoms of either high or low obstruction may predominate. $\mathrm{Ab}$ dominal pain is present in almost all cases, being either diffuse or predominantly concentrated at the place of the obstruction. Signs of peritoneal irritation appear when there are complications such as necrosis, perforation of the flap or hemoperitoneum. Digestive hemorrhaging occurs in a little over $40 \%$ of cases, apparent either through the upper or lower passage, with melena being the most frequent form. Other extra-hemorrhagic manifestations may also be present.

Simple radiography of the abdomen is insufficiently specific and may not show alterations or evidence of intestinal obstruction, such as gastric dilation, hydro-aero level, dilated and thickened small-bowel flaps and the "pile of coins" sign.

The barium x-ray, widely used until the $1980 \mathrm{~s}^{8}$, lost ground as a diagnostic tool method with the development of CT and US imaging tools. These methods are less invasive, more quickly and easily performed and offer a more adequate evaluation regarding the presence or absence of free liquid in the abdominal cavity, as well as the 
retroperitoneum. The combination of these two methods can offer $100 \%$ diagnostic accuracy, as demonstrated by Polat and Cols in 2003. ${ }^{27}$

Where CT is unavailable, US in combination with abdominal radiography may be sufficient to diagnose intramural hematoma. The indicative findings are tubular or circular images with absence of peristalsis and mucous compression enveloped by an anechoic halo corresponding to the thickened intestinal wall. The echogenecity varies according to stage of maturation of the hematoma. ${ }^{28}$

Despite the diagnostic viability of US, CT is the diagnostic exam of choice and has been proven to be extremely sensitive, showing alterations suggestive of the presence of hematoma in almost $100 \%$ of cases. ${ }^{10,12,14,15,18,27}$

Initially, CT should be performed without contrast, as this may mask the presence of intramural hemorrhage. The findings consist of a thickening of the wall greater than $1 \mathrm{~cm}$, with partial reduction to total obstruction of the passage, the "pseudo-kidney" and "coiled spring" signs, generally, in short segments (average $23 \mathrm{~cm}$ ) with profuse dilation of the flaps. ${ }^{18}$ In the first ten days, hyperdensity is seen, varying from 50 to 80 Hounsfield units depending on the time elapsed between the onset of the event and the exam; ${ }^{22,28}$ it then evolves towards hypodensity, with re-absorption and resolution of the radiological condition in a few weeks ${ }^{(6)}$. Particular attention should be given to the interpretation of the thickened intestinal wall, which occurs in other afflictions, such as inflammatory, infectious and neoplasic diseases as well as intestinal ischemia. ${ }^{22}$ Currently, in the absence of anatomopathological confirmation, the diagnosis is considered confirmed with the presence of clinical and radiological findings combined with resolution of the condition following control CT. ${ }^{(18)}$

Due to the rarity of this entity, there are no studies that contain sufficient evidence to standardize treatment. There are only case reports of this condition in the literature, the largest of which includes eight patients using oral anticoagulation. $^{29}$

The appearance as acute obstructive abdomen may lead to operative treatment, although the use of US and CT in diagnosis has contributed to changes in conduct. Surgical exploration, which was previously used as a diagnostic method, has now become primarily a treatment method reserved for cases with complications that include intra-abdominal hemorrhage, suspected ischemia with or without perforation and peritonitis due to diagnosis doubt or to late complications. Clinical treatment should be instituted in the absolute majority of cases.

The first measure is the immediate suspension of oral anticoagulants, and when possible, of medications used to enhance anticoagulation. Clinical treatment consists of gastric decompression in the presence of vomiting, the correction of electrolyte disturbances, vitamin $\mathrm{K}$ administration and the transfusion of fresh frozen plasma and blood when indicated. Total parenteral nutrition may be necessary in cases of prolonged fasting. In this review, we found one case treated with prothrombin, proconvertin, factor $\mathrm{X}$ and globulin B anti-hemophiliacs and heparin. ${ }^{30}$

In cases where there are no complications and surgery is unnecessary, resolution tends to occur within a few days. The recurrence of bleeding, as well as late sequelae such as stenosis, is unusual. Death during the presence of an intramural hematoma has occurred as a result of secondary complications such as digestive hemorrhage due to ulcer. ${ }^{28}$

\section{CONCLUSION}

Given the rarity of cases and the high incidence of painful and obstructive abdominal syndromes caused by highly prevalent affections often seen in emergency services, the diagnostic possibility of spontaneous intestinal intramural hematoma should be remembered when there are indications suggesting coagulation or other hemorrhagic manifestations, mainly in patients who use oral anticoagulants. Non-invasive investigative methods offer high accuracy, permitting the adoption of clinical procedures that avoid unnecessary surgery, with complete resolution of the condition in a short period in nearly $100 \%$ of cases.

\section{REFERENCES}

1. Brewer RJ, Golden GT, Hitch DC. Abdominal pain: An analysis of 1000 consecutive cases in a hospital emergency room. Am J Surg. $1976 ; 131: 219-223$

2. Lohn, JWG, Austin, RCT, Winslet, MC. Unusual causes of small-bowel obstruction. J R Soc Méd. 2000;93:365-368.

3. Konan AV, Rajhi H, Chatti K, Mnif N, Salem A, Hamza R. Intramural small-bowel hematoma due to anticoagulant therapy: a radiologic study; with regard to two cases. Tunis Med. 2005;83:233-6.
4. Cappelli J, Lenaerts A, Lamy V, Ramdani B, Moisse R. Clinical case of the month. Intramural hematoma of the small intestine associated with anticoagulants, potentiated by interaction with cimetidine. Rev Med Liege. 1997;52:753-5.

5. Yoshida T, Takami M, Fujimoto T, Shimizu H, Ota T, Takada T et al. Intramural hematoma of the small intestine caused by anticoagulant therapy: report of a case. Nippon Shokakibyo Gakkai Zasshi. 1991;88:1588-92. 
6. Avent ML, Canaday BR, Sawyer WT. Warfarin-induced intramural hematoma of the small intestine. Clin Pharm. 1992;11:632-5.

7. Hsiao CW, Chao PC. Warfarin-induced intramural haematoma of the ileocecal valve with obstruction. ANZ J Surg. 2004;74(9):810-1.

8. Vinard JL, Bouchet C, Aubert H, Meullenet J, Ohanessian JH, Aubert $\mathrm{M}$ et al. Intramural hematomas of the small bowel (duodenum excluded) during long-term anticoagulant treatment. Report on 6 cases of which 2 required operation (author's transl). J Chir (Paris). 1981;118:307-14.

9. Faria J, Pessoa R, Hudson M, Vitoi S, Villela O, Torres J et al. Hematoma intramural duodenal como complicação de terapia anticoagulante com Warfarin: relato de caso e revisão da literatura. Radiol Bras. 2004;37:461-463.

10. Shah P, Kraklow W, Lamb G. Unusual complication of coumadin toxicity. Wis Med J. 1994;93:212-4.

11. Meier R, Wyss-Meyer H, Jubin E, Gyr K. Intramural hematoma of the duodenum as complication in anticoagulant therapy. Diagnosis and therapy. Schweiz Rundsch Med Prax. 1990;79:517-20.

12. Secil M, Ucar G. Spontaneous duodenal hematoma. J Emerg Med. 2004;27:291-3.

13. Giesbers AA, Voets AJ, de Smet HL, van Wilderen LJ. Intramural hematomas of the small intestine during use of oral anticoagulants. Ned Tijdschr Geneeskd. 1986;130:113-7.

14. Acea Nebril B, Taboada Filgueira L, Sanchez Gonzalez F, Freire Rodriguez D, Fraguela Marina J, Aguirrezabalaga Gonzalez J et al. Acute abdomen in anticoagulated patients. Its assessment and the surgical indications. Rev Clin Esp. 1995;195:463-7.

15. Rios R, Garaulet P, Rodriguez M, Leon C, Limones M. Spontaneous intramural hematoma of the small intestine. Cir Esp. 2005;78:275.

16. Fahrhoud S, Stephani SM, Bromberg SH. Pancreatite aguda devida a hematoma intramural do duodeno por uso de anticoagulante. Arq. Gastroenterol. 2001;38:53-56.

17. Liverud, K. Hematoma of the jejunum with subileus. Arch Radiol 1948;30:163; in aput Jones WR, Hardin WJ, Davis JT, Hardy JD. Intramural hematoma of the duodenum: a review of the literature and case report. Ann Surg. 1971;173:534-544.

18. Abbas MA, Collins JM, Olden KW. Spontaneous intramural small-bowel hematoma: imaging findings and outcome. AJR Am J Roentgenol. 2002;179(6):1389-94.
19. Bettler S, Montani S, Bachmann F. Incidence of intramural digestive system hematoma in anticoagulation. Epidemiologic study and clinical aspects of 59 cases observed in Switzerland (1970-1975). Schweiz Med Wochenschr. 1983;113(17):630-6.

20. Shaw PH, Ranganathan S, Gaines B. A spontaneous intramural hematoma of the bowel presenting as obstruction in a child receiving low-molecular-weight heparin. J Pediatr Hematol Oncol. 2005;27:55860.

21. Hill H, Deppe H, Huchzermeyer H, Dormann AJ. Duodenal ileus due to an intramural duodenal haematoma. Conservative therapy using a multiple lumen intestinal probe. Dtsch Med Wochenschr. 2005;130:924.

22. Lane MJ, Katz DS, Mindelzun RE, Jeffrey RB Jr. Spontaneous intramural small bowel haemorrhage: importance of non-contrast CT. Clin Radiol. 1997;52:378-80.

23. Dubois J, Guy F, Porcheron J. A pancreatic-induced intramural duodenal hematoma: a case report and literature review. Hepatogastroenterology. 2003;50:1689-92.

24. Dineen RA, Lewis NR, Altaf N. Small bowel infarction complicating rectus sheath haematoma in an anticoagulated patient. Med Sci Monit. $2005 \cdot 11:$ CS57-9.

25. de Mello G, Choma L, Santos HR, de Paola. Intraparietal hematoma of the small intestine as a complication of anticoagulant therapy. Presentation of 1 case with anatomo-pathological confirmation. Hospital (Rio J). 1963;63:121-7.

26. Judd DR, Taybi H, King H. Intramural hematoma of the small bowel; a report of two cases and a review of the literature. Arch Surg. 1964;89:527-35.

27. Polat C, Dervisoglu A, Guven H, Kaya E, Malazgirt Z, Danaci M et al Anticoagulant-induced intramural intestinal hematoma. Am J Emerg Med. 2003;21:208-11.

28. Lorente-Ramos RM, Santiago-Hernando A, Del Valle-Sanz Y, ArjonillaLopez A. Sonographic diagnosis of intramural duodenal hematomas. J Clin Ultrasound. 1999;27:213-6.

29. Abbas MA, Collins JM, Olden KW, Kelly KA. Spontaneous intramural small-bowel hematoma: clinical presentation and long-term outcome. Arch Surg. 2002;137:306-10

30. Walter P, Scheffler P, Heinz J. Overdosage of anticoagulants as a rare cause of acute abdomen. Chirurg. 1984;55:532-3. 